\title{
ARTÍCULOS
}

Recibido 09.12.2013. Aprobado 12.08.2014

Evaluado por el sistema double blind review. Editor Científico: Mário Sacomano Neto

DOI: http://dx.doi.org/10.1590/So034-759020150107

\section{GESTIÓN DE RECURSOS INTANGIBLES EN INSTITUCIONES DE EDUCACIÓN SUPERIOR}

\author{
Gestão de recursos intangíveis em instituições de ensino superior \\ Management of intangible resources at higher education institutions
}

\section{RESUMEN}

Las organizaciones se desenvuelven en entornos exigentes y competitivos creados por demandas de las sociedades y marcados por la crisis financiera mundial a la que no escapan las Instituciones de Educación Superior (IES), que están orientadas a satisfacer las necesidades del entorno y a fortalecer el flujo de información y colaboración con la sociedad. En este contexto se plantea una investigación de tipo exploratorio y descriptivo, que se considera como un primer intento por desarrollar y probar empíricamente los aspectos teóricos existentes sobre el tema. El objetivo es determinar las influencias simultáneas que ejercen los recursos intangibles sobre la gestión estratégica en las IES. Estas relaciones se evaluaron con un análisis de estructuras de covarianzas que requirió la construcción y validación de un sistema de medida adecuado para cada una de las variables objeto de estudio. Dicho análisis arrojó como resultado que sólo dos de las cuatro hipótesis planteadas son significativas, loque demuestra que los recursos intangibles Gestión del conocimiento y Reputación Organizacional, son los que influyen directamente en los procesos de gestión estratégica que realizan las IES. A partir de estos descubrimientos se dispone de referentes empíricos sobre la Gestión de Recursos Intangibles, destacando el hecho que la gestión estratégica en las IES puede mejorar sustancialmente a través de la implementación de procesos que involucren el manejo adecuado de los intangibles que poseen.

PALABRAS CLAVE | Gestión estratégica, Instituciones de Educación Superior (IES), recursos intangibles, capital intelectual, Modelo de Ecuaciones Estructurales (MEE).

\section{RESUMO}

As organizações se desenvolvem em ambientes exigentes e competitivos criados pelas demandas das sociedades e marcados pela crise financeira mundial na qual não escapam as Instituições de Educação Superior (IES), que estão orientadas para satisfazer as necessidades do ambiente e para fortalecer o fluxo de informação e colaboração com a sociedade. Neste contexto, propõe-se uma pesquisa do tipo exploratória e descritiva, como uma primeira tentativa de desenvolver e provar empiricamente os aspectos teóricos existentes sobre o tema. $O$ objetivo é determinar as influências simultâneas que os recursos intangiveis exercem sobre a gestão estratégica nas IES. Estas relações serão avaliadas com uma análise de estruturas de covariâncias requerida da construção e validação de um sistema de medida adequado para cada uma das variáveis do objeto de estudo. Como resultado da análise, somente duas das quatro hipóteses propostas são significativas, o que demonstra que os recursos intangiveis gestão do conhecimento e reputação organizacional são os que influem diretamente nos processos de gestão estratégica que realizam as IES. A partir destas descobertas, dispõe-se de referências empíricas sobre a gestão de recursos intangíveis, destacando o fato de que a gestão estratégica nas IES pode melhorar substancialmente por meio da implementação de processos que envolvem o manejo adequado de todos os recursos intangíveis disponíveis.

PALAVRAS-CHAVE I Gestão estratégica, Instituições de Ensino Superior (IES), recursos intangíveis, capital intelectual, Modelo de Equações Estruturais (MEE).

YILSY MARÍA NÚÑEZ GUERRERO yilsy.nunez.guerrero@alumnos.upm.es Doctora en Administración de Empresas por la Universidad Politécnica de Madrid, Escuela Técnica Superior de Ingenieros Industriales - Madrid, España

\section{CARLOS RODRÍGUEZ MONROY} crmonroy@etsii.upm.es Profesorde la Universidad Politécnica de Madrid, Escuela Técnica Superior de Ingenieros Industriales, Departamento de Ingeniería de Organización, Administración de Empresas y Estadística - Madrid, España

\section{ABSTRACT}

The organizations are developed in challenging and competitive environments, created by demands of societies, and marked by the global financial crisis that extends even to the Institutions of Higher Education (IHEs), which are aimed at meeting the needs of the environment and at strengthening the flow of information and collaboration with the society. In this context, an exploratory and descriptive investigation, which is considered as a first attempt to develop and empirically test the existing theoretical aspects of the subject arises. The objective is to determine the simultaneous influences exerted by intangible resources on strategic management in IHEs. These relationships were assessed by means of an analysis of covariance, which required the construction and validation of an appropriate system of measurement for each of the variables under study. Such analysis showed, as a result, that only two of the four hypotheses are significant, demonstrating that the intangible resources, namely Knowledge Management and Organizational Reputation, are those that directly affect strategic management processes, undertaken by the IHEs. From these findings, one has empirical references on the Management of Intangible Resources, thus highlighting the fact that the strategic management in IHEs can be substantially improved by means of the implementation of processes that involve the proper management of intangible assets that they have.

KEYWORDS I Strategic Management, Institutions of Higher Education (IHES), intangible assets, intellectual capital, Structural Equation Model (SEM). 


\section{INTRODUCCIÓN}

Debido a que la crisis financiera mundial iniciada a finales de 2007, es una crisis continuada, según Krugman (2012), la economía se está enfrentando a nuevos retos. En consecuencia, se hace necesario que las "naciones ricas en recursos, talento y conocimientos” fortalezcan estos elementos para así lograr la pronta recuperación.

En este contexto, Secundo, Margherita, Elia, \& Passiante (2010), plantean que las IES deben permanecer en concordancia con el compromiso de proporcionar las herramientas de conocimiento y transmitir la información necesaria sobre los requerimientos disciplinarios, tecnológicos y actividades de $I+D$, que se traducen en una mejora de la calidad de vida de la sociedad.

Consecuentemente, este tipo de organizaciones se ven obligadas a fomentar sus capacidades de crear recursos intangibles requiriendo a su vez de estos mismos recursos para poder llevar a cabo con éxito su función mediante la facilitación del proceso educativo, fortaleciendo de esta manera las ventajas estratégicas que le proporcionan los recursos y las capacidades intrínsecas, (Viedma, 2007; Morales, Medina, \& Álvarez (2003).

No obstante, desde hace más de dos décadas, se ha venido desarrollado el cuerpo de teorías sobre el tema de los intangibles (Barney, 1991; Grant, 1991; Bontis, 1998; Edvinsson \& Malone, 1997; European Commission, 2006, 2011; Kaplan \& Norton, 2004; Seemann, Delong, Stucky, \& Passiante, 2000; Sveiby, 1997, 2010) las cuales se limitan a explicar los elementos que definen a los intangibles organizacionales solo nombrándolos de diferentes maneras. Además se incorpora la gestión estratégica para visualizar y dirigir las acciones necesarias a través de la combinación de los recursos tangibles e intangibles para lograr una mejor posición competitiva y generar valor para las organizaciones (Bueno, 1998; Petty \& Guthrie, 2000; Andriessen, 2004, Sánchez \& Heene, 2004).

Por lo tanto, el desarrollo investigativo sobre intangibles todavía es escaso y se caracteriza por presentar potenciales intentos de generalizar criterios y estándares, pero adolece de heterogeneidad, que más que contribuir a unificar, dispersa los diferentes estudios sobre el tema (Secundo et al., 2010).

Aunado a lo anterior se destaca que se sabe poco del significado que tienen los intangibles asociados a la dirección estratégica para las IES. Aun cuando está extendida la creencia que los intangibles generan valor, no son considerados prioritarios en la gestión de las mismas (Kristandl \& Bontis, 2007; Cañibano \& Sánchez, 2008).
Asimismo, en ningún caso se han analizado de manera simultánea las influencias de los recursos intangibles en la gestión estratégica que realizan las IES, lo que constituye una brecha en el conocimiento.

A esto hay que agregar que los estudios antes citados carecen de referentes de validez de las escalas de medición, aunque existen estudios que han desarrollado instrumentos que permiten captar algunas dimensiones de manera aislada considerando los intangibles de forma separada (Alvarado \& Schlesinger, 2008; Villafañe, 2003; Capriotti 2007; Cañibano \& Sánchez, 2004).

Por lo antes planteado se considera necesario profundizar en el conocimiento, de los diferentes tipos de recursos intangibles que poseen las IES, y en la influencia que ejercen en la gestión estratégica en estas instituciones. La presente investigación tiene como objetivo determinar la influencia que los recursos intangibles tienen sobre la gestión estratégica que realizan las IES.

Con el fin de disponer de evidencias empíricas que sustenten las posibles relaciones de causalidad que subyacen entre las variables en estudio, se utiliza la técnica de Modelo de Estructuras de Covarianzas (Structural Equation Modeling SEM) y se plantea una investigación que se basa en el enfoque de la teoría de recursos y capacidades, la cual contribuye a considerar a los recursos intangibles como parte esencial del subsistema de recursos de las IES, y de esta forma analizar la influencia que tienen sobre la gestión estratégica.

A continuación se presenta el desarrollo, los resultados y las principales conclusiones, lo que permite identificar cuáles de los recursos intangibles influyen en la gestión estratégica en las IES.

\section{PLANTEAMIENTOS TEÓRICOS E HIPÓTESIS}

Las teorías sobre intangibles se originan en la década de los noventa del siglo XX al empezar a estudiarse a los intangibles como recursos estratégicos. Se formaron corrientes de investigación enmarcadas en diferentes disciplinas (Economía, Gestión de Empresas, Sociología y Finanzas, entre otras), No obstante, es la teoría de recursos y capacidades (TRC) la que proporciona los postulados básicos para el desarrollo del cuerpo de teórico sobre intangibles.

Esta teoría, plantea que las organizaciones son diferentes entre sí en función de los recursos y capacidades que poseen en un momento determinado (Barney, 1991). Adicionalmente se afirma que la función principal de la dirección de una 
empresa es la maximización de su valor a través de la utilización de los recursos y capacidades existentes (Grant, 1996).

Asimismo, resalta la importancia que tienen los recursos intangibles de las organizaciones. Es por ello, que las diferentes corrientes tratan, en primer lugar, de ordenar de forma estructurada los intangibles y, en segundo lugar buscar indicadores que permitan medirlos, para lograr comparar la situación de la organización con otras de referencia (Brooking, 1996; Edvinsson \& Malone, 1997; Lev, 2003; Sveiby, 1997; Bueno, 2003; Sánchez, 2008).

Sin embargo, al analizar las investigaciones generadas en el campo objeto de estudio, se puede observar que existe una amplia terminología toda ella dirigida a aspectos relativos a los intangibles. Por lo que, se evidencia falta de consenso sobre los términos y sus definiciones.

Los primeros intentos por definir los recursos intangibles se dieron en la época de los 9o, esbozando que los mismos estaban dados por la ecuación que refleja la diferencia en el valor de mercado de una organización con respecto a su valor en libros. A esta diferencia se le llama capital intelectual.

Sin embargo, según Barney (1991) se considera recurso a todos los activos, capacidades, procesos organizativos, conocimientos, entre otros, controlados por una empresa, que permiten la implantación de estrategias que mejoran la eficiencia y la eficacia de la misma. En consecuencia, en las organizaciones se evidencia la existencia de activos por un lado y recursos intangibles por otro, que no son la misma cosa. Los activos intangibles según Nomen (2005) son los que carecen de propiedad física y son susceptibles de ser evaluados con criterios económicos financieros. Los recursos se diferencian de los activos en que no se pueden cuantificar en valores contables, por lo que las empresas no pueden garantizar su control (Galbreath \& Galvin. 2006). Por lo tanto, a efectos de esta investigación, los recursos intangibles son un concepto más amplio que el de activo intangible.

No obstante, se consideran recursos intangibles a todos aquellos recursos de las organizaciones que carecen de existencia física, que son potencialmente utilizables, que son renovables luego de usarlos y que no disminuyen sino que aumentan en cantidad y calidad mientras son usados, además de poder ser usados de manera simultánea, y presentar un elevado grado de especialización (Diefenbach, 2006).

Esta definición está en concordancia con lo expuesto por Barney (1991), sobre las características que deben poseer los recursos estratégicos de las organizaciones para poder generar una ventaja competitiva. Las mismas se refieren al grado de escases, valor, no imitabilidad y la no sustitución.
Debido a la importancia que estos recursos aportan para el éxito de las organizaciones, Villafañe (2005) expone que los recursos intangibles empresariales son en definitiva: la marca, la gestión del conocimiento, la responsabilidad social empresarial y la reputación corporativa.

En este mismo sentido, Villalonga (2004) afirma que la TRC hace hincapié en la importancia de los recursos intangibles como la clave para la sostenibilidad. Y cita la definición aportada por Itami (1987) que sugiere que los intangibles, que pueden estar dados por una tecnología en particular, el nombre de marca, la reputación y la cultura de la empresa, son de gran valor para la capacidad competitiva de la misma. Y frecuentemente son la única fuente real de ventaja competitiva que puede ser sostenido en el tiempo.

Asimismo, según Grant (1991) los recursos intangibles están referidos a la marca, la reputación, la confianza de los clientes, la información, patentes y Know-how. Todas estas definiciones convergen en señalar diversos elementos que poseen las organizaciones y están enmarcados en las características básicas de intangibilidad (escasa, valiosa, difícil de imitary de sustituir).

Otros autores pretenden describir la naturaleza de los recursos intangibles realizando categorizaciones de los mismos sin antes tratar de definirlos, en este sentido Cañibano, García-Ayuso, y Chaminade (1999), tras una exhaustiva revisión bibliográfica concluye que existe un cierto consenso en la naturaleza económica de los intangibles, mas no en su clasificación.

Así como se muestra en el Cuadro 1, se considera que se habla de intangibles cuando estamos en presencia de alguno de los siguientes términos (propiedad intelectual, activo intangible, capital intelectual, capital de conocimiento).

También los términos usados incluyen los siguientes conceptos: intangibles, activos intangibles, capital intangible, recursos intangibles, capital intelectual y propiedad intelectual (Kaufmann \& Schneider, 2004).

Cuando se considera, la definición de intangibles a partir de categorías, es posible identificar diversidad de términos y ubicar las diferentes clases intangibles. En el Cuadro 2 se puede visualizar alguna de las categorías de intangibles que existen a la fecha destacando que sólo se muestran las que están asociadas a los términos antes mencionados.

Es así que, los términos activos intangibles y capital intelectual empiezan a ser utilizados en la literatura sobre el tema de manera indiferente (Sánchez, 2008) para referirse a ese cúmulo de capacidades, actividades o recursos que están presentes en las organizaciones y que las hace diferentes de otras del mismo sector por su capacidad de generar valor. 


\section{CUADRO 1. Términos que se relacionan con intangibles}

\begin{tabular}{l|l}
\hline Término & Definición \\
\hline Propiedad Intelectual & $\begin{array}{l}\text { Tiene que ver con las creaciones de la mente: las invenciones, las obras literarias y artísticas, los } \\
\text { símbolos, los nombres, las imágenes y los dibujos y modelos utilizados en el comercio. }\end{array}$ \\
\hline Activo Intangible & $\begin{array}{l}\text { Aquel recurso generador de futuros beneficios económicos, que está en posesión de la empresa y que, } \\
\text { además, carece de sustancia física y tiene larga duración. } \\
\text { SFAS número 3 (FASB, 1977) y SFAS número 142 (FASB, 2001). }\end{array}$ \\
\hline Capital Intelectual & $\begin{array}{l}\text { Acumulación de conocimiento que crea valor o riqueza cognitiva poseída por una organización, } \\
\text { compuesta por un conjunto de activos de naturaleza intangible o recursos y capacidades basados en } \\
\text { conocimiento, que cuando se ponen en acción, según determinada estrategia, en combinación con el } \\
\text { capital físico o tangible, es capaz de producir bienes y servicios y de generar ventajas competitivas o } \\
\text { competencia esenciales para la organización en el mercado (PULIDO, 2007). }\end{array}$ \\
\hline Capital de Conocimiento & $\begin{array}{l}\text { Capital de conocimiento es el saber que los resultados de la experiencia, información, conocimiento, } \\
\text { aprendizaje y habilidades de los empleados o individuales de una organización o grupo. }\end{array}$ \\
\hline
\end{tabular}

\section{CUADRO 2. Categorías de Capital Intelectual}

\begin{tabular}{l|l}
\hline Propuesto por: & Clasificación \\
\hline Proyecto MERITUM & Recurso y Actividades, Capital Humano, Capital Relacional, Capital Estructural. \\
\hline Villafañe (2005) & Marca, Gestión del Conocimiento, Responsabilidad Social, Reputación Corporativa. \\
\hline $\begin{array}{l}\text { Diefenbach (2006), De Gregori } \\
(2002)\end{array}$ & Capital Humano, Capital Relacional, Capital Cultural, Capital Legal, Capital Informativo, Capital Arraigado. \\
\hline
\end{tabular}

Por todo lo antes expuesto se puede decir que los estudios realizados hasta la fecha sobre intangibles presentan diferentes formas de definirlos, categorizarlos y gestionarlos, haciendo especial énfasis en la gestión de intangibles como herramienta de gerencia que utiliza indicadores para medir y controlar estos recursos en las organizaciones.

En este orden de ideas las IES son unas de las principales organizaciones que están prestando atención al manejo de sus recursos tanto tangibles como intangibles, lo que se evidencia en la cantidad de investigaciones desarrolladas como iniciativa de grandes instituciones, como el World Bank (2011), la OCDE (2010) y EU (2011) entre otras.

La gestión de los intangibles y del capital intelectual en las IES es de importancia decisiva, aún más que en otro tipo de organizaciones, debido a que las metas de una universidad son la producción y la difusión del conocimiento, por lo que las mismas se dedican a invertir principalmente en recursos humanos, es decir en activos intangibles (Cañibano \& Sánchez, 2004).

En este orden de ideas, una de las misiones de las IES según Secundo et al.,. (2010) es formar el capital humano del futuro, capaz de estimular la innovación organizativa y tecnológica así como aumentar la red de relaciones entre la academia y el sector empresarial. Sin embargo, para lograr las metas o misiones de las IES, es necesario gestionar los recursos intangibles de manera adecuada, de forma tal que aumenten su capacidad innovadora y generen valor.

Por este motivo, es primordial que las IES se conozcan a sí mismas, comprendiendo completa y profundamente los recursos y capacidades que poseen (Grant, 1996). Entre ellas se encuentran: marcas, conocimiento, contactos comerciales, procedimientos eficientes, responsabilidad social corporativa, reputación corporativa, capacidades del capital humano, know-how de la organización, conocimiento tecnológico, contactos con clientes y proveedores) (Wernerfelt, 1984; Accenture, 2012) centrando su atención en la identificación, desarrollo, protección y despliegue de los recursos antes mencionados.

Como se ha expuesto anteriormente, dada la diversidad de disciplinas que han abordado el tema de los intangibles, se puede encontrar que diferentes autores pretenden describir la naturaleza de los recursos intangibles realizando categorizaciones de los mismos sin antes tratar de definirlos (Kristandl \& Bontis, 2007).

Entre las clasificaciones más relevantes se encuentran las propuestas de Edvinsson y Malone (1997), Ross, Ross, Edvinson, y Dragonetti (1998), Diefenbach (2006), Kaplan y Norton (2007), Sveiby (2010). Estas clasificaciones no se discuten en la presente investigación. 
Sin embargo, sí se expone la categorización propuesta por Villafane (2005) que clasifica a los recursos intangibles en tres tipos: los activos no financieros de las organizaciones, los asociados al conocimiento y los que son resultado de una relación estable y eficaz con sus stakeholders. Agrega, además, que la práctica de la gestión de los intangibles organizacionales, está dada en concreto por estos cuatro recursos intangibles: la Marca, la Gestión del Conocimiento, la Responsabilidad Social Corporativa y la Reputación Corporativa.

Este autor fundamenta sus teorías en que los intangibles provienen de la dirección estratégica, debido a que la gestión del conocimiento y todos los intangibles crean procesos de generación de valor y resalta que estos recursos sólo tienen relevancia con la comunicación, orientada a fortalecer la imagen, marca, responsabilidad social y reputación de las propias compañías. Cabe resaltar que la perspectiva propuesta por el autor está alineada con el marketing.

Estos enunciados son perfectamente aplicables a la IES, que aunque su función principal no sea conseguir beneficios económicos sino lograr la misión de educar, formar y realizar investigaciones (UNESCO, 1998), son instituciones que se han visto en la necesidad de adoptar los principios de la dirección estratégica para manejar su proceso y recursos de manera eficiente (Sánchez, Elena, \& Castrillo, 2006). Estas instituciones desde la perspectiva empresarial están ubicadas en el sector de servicios, puesto que proporcionan a la sociedad productos intangibles (enseñanza e investigación) Bricall (2000). Aunado a esto, la educación es un proceso basado principalmente en la comunicación, por lo que es lógico que la comunicación sea parte esencial de los procesos de gestión en las IES.

En la educación superior, según López (2012), se identifican tres elementos básicos de la gestión: 1. La dirección de las instituciones, 2. La gestión académica y 3. La gestión eficiente de los recursos. Por lo que es de esperar que las IES, cuenten con un eficaz sistema de gobierno que les proporcionen los procedimientos de soporte necesarios para el logro de sus objetivos estratégicos. Además tener la capacidad de ser flexibles para adaptarse a los cambios, desarrollar intangibles intensivos en tecnología, fomentar la utilización de información y modernizar los procesos académicos con alto grado de innovación, logrando estructuras curriculares que permitan comparar resultados y hacerlos compatibles (Gacel-Ávila, 2011; Varela-Candamio \& García-Álvarez, 2012).

En consecuencia, se ven obligadas a orientarse hacia la gestión eficiente de los recursos para que los mismos contribuyan sustantivamente al mejoramiento permanente del talento humano, de la infraestructura física y tecnológica y de la gestión del servicio que proporcionan. A esto se suma el hecho que las
IES deben optimizar la utilización de los conocimientos generados, para contribuir al beneficio social y económico de las regiones (Rodríguez-Ponce, Pedraja-Rejas, Araneda-Guirriman, \& Rodríguez-Ponce, 2013).

Existen en la literatura sobre el tema algunas investigaciones (Leitner, 2004; Meritum, 2002; Cañibano \& Sánchez, 2009; Bustos, 2007; Funes, 2010; Sánchez et al.,. 2006; Secundo et al.,. 2010; López, 2012), que exponen modelos de gestión del capital intelectual para universidades. Sin embargo, todos ellos se enfocan en comunicar a través de reportes periódicos cuáles son los elementos de creación de valor asociados a cada intangible, sin tener en cuenta las interrelaciones que existen entre los diferentes intangibles. Además proponen conjuntos de indicadores en el mejor de los casos, pero sin el respectivo rigor estadístico.

En general las características del sistema universitario están dadas por lo heterogéneo del tamaño de las instituciones, el tipo (pública o privada) y el entorno que puede resultar altamente competitivo, en cuanto a recursos humanos, tecnológicos y financieros, además de ser un sector indiferenciado (Villafañe, 2013).

Sin embargo, es indudable que las teorías sobre intangibles proporcionan una base para la compresión del desempeño organizacional, además abordan diferentes recursos al mismo tiempo, junto con dispositivos de comunicación y sistemas de control de gestión, lo que los hace factores claves en el proceso de creación de valor de un organismo público (Sánchez et al.,. 2006).

Por lo tanto, la clasificación de recursos intangibles realizada por Villafañe (2005), así como la definición de los aspectos relevantes de la gestión de intangibles son los elementos que se han tomado como base para la realización de esta investigación, debido a la importancia de los intangibles para las IES, por ser elementos intensivos en conocimiento y capacidades, los que los convierte en recursos claves para la generación de valor, traducidos en estas instituciones en innovaciones. (Sánchez et al., 2006).

\section{Hipótesis}

A continuación se muestra el sistema de hipótesis (que corresponden con cada variable que se ha considerado) que están siendo sometidas a confirmación para determinar el conjunto de relaciones causales que se presentan entre los factores que determinan los tipos de recursos intangibles (Gestión del Conocimiento, Marca, Reputación Corporativa y Responsabilidad Social) que se utilizan como variables independientes, con la gestión estratégica que realizan las IES, que ha tomado como variable dependiente. 
Por lo tanto, la gestión de recursos intangibles según señala Villafañe (2005) no supone una gestión aparte de la tradicional, sino que está integrada en la dirección estratégica y alineada con la filosofía de gestión de la organización y presenta cuatro aspectos relevantes: 1 . Gestión orientada a la estrategia que incluye los recursos intangibles. 2. Posesión de un sistema de valoración de los recursos intangibles 3. Integración de la gestión de recursos intangibles en la cadena de valor. 4. Implementación de estrategias de comunicación de los recursos intangibles.

Así se tiene que los recursos intangibles necesitan de los procesos de comunicación para poder ser manejados eficientemente de forma tal que se puedan establecer políticas de gestión estratégica para los mismos en pro de la eficiencia organizacional. Como asegura Bueno (1998) las decisiones estratégicas que tome una organización que sabe aprender aprende estarán enfocadas a maximizar los intangibles que posee.

Partiendo entonces de la clasificación de intangibles propuesta por Villafañe (2005), a continuación se presenta la definición de cada uno de los recursos intangibles estudiados:

- Gestión del Conocimiento es el conjunto de actividades donde se potencian los tipos de conocimiento (tácito y explicito) y las formas de almacenarlos y transferirlos con los proceso de construcción de más conocimiento (Nonaka \& Takeuchi, 1995), teniendo en cuenta que el conocimiento explícito es el conocimiento transmisible de la organización, es decir, aquel que cuenta con la posibilidad de poder ser intercambiado al estar representado mediante palabras o números.

El conocimiento tácito, por su parte, es el que se encuentra arraigado en los individuos, en las acciones y experiencias, así como en sus ideales, valores y emociones y por lo tanto es de difícil transmisibilidad. Entonces, es difícil de imitar, sustituir, transferir y es raro (Ambrosini \& Bowman 2001).

Según Saíz-Bárcenas, Díez-Pérez, Manzanedo Del Campo, \& Rodríguez-Monroy, (2013) el conocimiento gestionado de forma intencionada genera aprendizaje a partir de las experiencias y perfeccionamiento de los procedimientos, formándose así un ciclo continuo que genera beneficios económicos, sociales y financieros.

Por lo cual, según Villafañe (2013) el talento, en este caso denominado conocimiento, es una herramienta clave para crear competitividad y diferenciación en las organizaciones, determinando los factores que inciden directamente en la calidad de los profesionales y el clima laboral, además de los factores que tienen que ver con personalidad, desempeño, actitud, experiencia entre otros.

Así se tiene que si la organización procura gestionar el conocimiento que poseen los individuos que la conforman en forma de talento, esto contribuirá a: potenciar los demás recur- sos de la institución, generar el valor a través del conocimiento, maximizar los aportes de todos los integrantes de la IES, traducido esto en mayor compromiso y mayor productividad y el logro de la transferencia de conocimientos a la sociedad.

Se plantea entonces la siguiente hipótesis:

$\mathrm{H}_{1}$ : La Gestión Estratégica de las IES está influenciada directa y positivamente por la Gestión del Conocimiento.

- 2. La Marca refleja de una forma poderosa lo que constituye la generación de valor para cualquier organización, expresa la identidad y los valores asociados a la IES traducidos en compromisos y promesas verificables (Villafañe \& Asociados, 2013). En general resume todo lo que el cliente final es capaz de percibir de una organización. Es considerada un recurso estratégico que vincula la organización con el entorno. Los atributos que la definen en el caso de las instituciones de educación superior son: reconocimiento social, preocupación por la sociedad, logro de objetivos, actitudes, vínculo emocional y asociaciones, todos ellos con el fin de diferenciar la organización (Aaker, 2004; Kotler, 2000; Curubeto, 2007; Capriotti, 2009). Por ello se plantea:

$\mathrm{H}_{2}$ : La Gestión Estratégica de las IES está influenciada directa y positivamente por la Marca.

- 3. Reputación Organizacional de las IES es el reconocimiento de su excelencia por parte de sus grupos de interés, en función de su comportamiento académico, la satisfacción de las expectativas de acuerdo a los compromisos adquiridos, que se encuentran expresados en la visión, misión y valores (Villafañe, 2013). Los atributos que la definen son: la imagen, la identidad, la cultura organizacional, la percepción de los stakeholders, la actuación y gestión pasada de la organización, las expectativas generadas y las comparaciones con sus competidores. Las variables básicas de la reputación están dadas por los valores reputacionales de las IES, que son los siguientes: 1 . Calidad docente que se evidencia en el perfil del profesorado, oferta docente y composición del alumnado. 2. Excelencia investigadora, compuesta por captación de fondos públicos para investigación, porcentaje de servicios de investigación y publicaciones científicas. 3. Gestión académica marcada por la profesionalización de la gestión, recursos e infraestructura e inserción social. (Dowling, 1994; Fombrun, 1996; Villafañe, 2005, 2013; Capriotti, 2009). Se plantea la siguiente hipótesis.

$\mathrm{H}_{3}$ : La Gestión Estratégica de las IES está influenciada directa y positivamente por la Reputación Organizacional. 
- 4. La Responsabilidad Social es la articulación de las diversas partes de la institución en un proyecto de promoción social de principios éticos y de desarrollo social equitativo y sostenible, para la producción y transmisión de saberes responsables y la formación de profesionales ciudadanos igualmente responsables. Se considera la responsabilidad social como los comportamientos éticos, y responsables que potencian y generan valor para las organizaciones, por lo cual se considera un recurso intangible que debe ser gestionado junto con los antes descritos (Villafañe \& Asociados, 2013). Debe estar integrada a la estrategia institucional en línea con los objetivos, debido a que constituye una forma de vincularse con la sociedad. Sus principales atributos en el entorno de las IES son: gestión ética y calidad de vida institucional, gestión medioambiental responsable, formación académica socialmente responsable, investigación socialmente útil y gestión social del conocimiento responsable (Alvarado \& Schlesinger, 2008; Hill, 2004; Dahlsrud, 2008; Vallaeys, 2007). La hipótesis correspondiente se plantea de la siguiente manera:

$\mathrm{H}_{4}$ : La Gestión estratégica de las IES está influenciada directa y positivamente por la Responsabilidad Social.

Desde el punto de vista de la categorización de propuesta por Villafañe (2005), cabe destacar que al considerar los intangibles como recurso estratégicos se hace evidente que el tipo de gestión que los enmarca de mejor manera es la gestión estratégica.

Por lo que, a partir de estas cuatro hipótesis se diseña el modelo teórico que se representa en la Figura 1.

Figura 1. Modelo Teórico de la influencia de los tipos de Recursos Intangibles en la Gestión Estratégica de las IES.

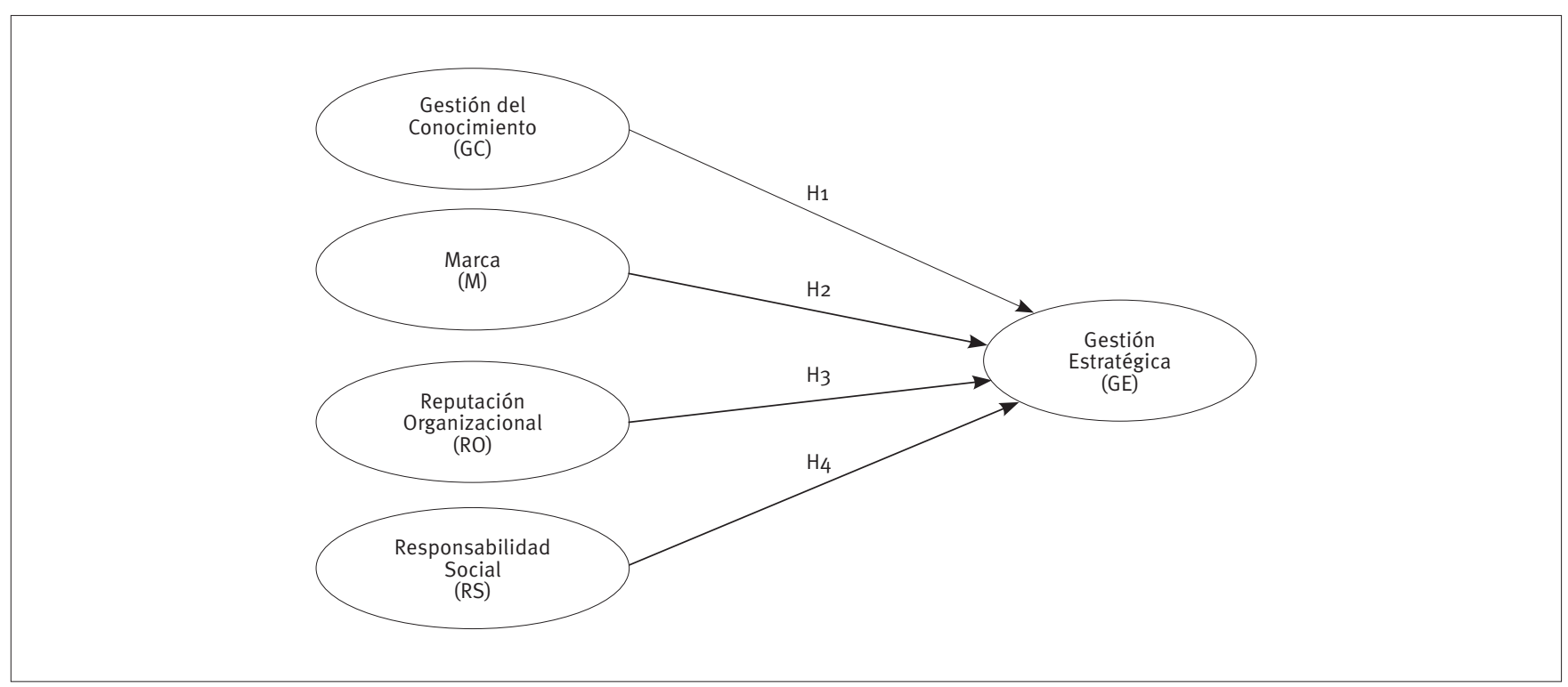

\section{METODOLOGÍA}

A fin de cumplir con el objetivo propuesto se plantea una investigación no experimental de tipo exploratoria, descriptiva. Se emplea una muestra de 247 profesores mayoritariamente pertenecientes a los consejos directivos de las IES, que se extrajo de la base de datos que gestiona el Consorcio de Escuelas de Ingeniería de Latinoamérica y del Caribe (LACCEI) y el Vicerrectorado de Asuntos Internacionales de la Universidad Politécnica de Madrid (UPM).

Se aplica un instrumento tipo encuesta online construido a partir de los referentes teóricos sobre el tema ( $a d$ hoc), en este caso se utilizó escala tipo likert, con 5 niveles de respuesta.
Para obtener la muestra de ítems relevante que sean capaces de medir cada uno de los constructos (Clark \& Watson, 2003), el cuestionario se organiza en base a un conjunto de ítems que se somete a análisis de expertos para evaluar la validez de contenido (Smith, Fischer, \& Fister 2003). La puesta en marcha de la encuesta se difundió mediante correo electrónico.

Respecto al grado de participación, de un total de 280 correos enviados, después de manejar los datos faltantes, el número de cuestionarios adecuados fue de 247 , repartidos en 53 IES. Ubicadas en Argentina, Brasil, Colombia, Chile, Costa Rica, Ecuador, España, México, Panamá, Perú, Portugal, Puerto Rico, República Dominicana y Venezuela. Los datos obtenidos se analizaron con el software estadístico SPSS V.20. AMOS V.20. 
Para proceder con el estudio y comprobar en qué medida los datos de la muestra apoyan los efectos directos e indirectos entre variables observadas del modelo teórico planteado, fue necesario establecer el modelo de medida del mismo.

Por lo que, para la medición de cada uno de los constructos correspondientes a los tipos de recursos intangibles (variables independientes) y la de la gestión estratégica de las IES (variable dependiente). Se construyen y validan las escalas para cada constructo, las cuales son independientes y se ubican dentro del cuestionario con un número de ítems que tienen la capacidad de poner de manifiesto las diferencias existentes entre los individuos. Con ello, se consigue maximizar la varianza del test (Carretero-Dios \& Pérez, 2005; Nunnally \& Bernstein, 1995).
Sin embargo, se debe tener presente que el conjunto de ítems además de presentar consistencia interna, que se refiere a que los ítems estén intercorrelacionados, también deben presentar homogeneidad, que se verifica cuando los ítems que miden el constructo son representativos de ese único componente.

Por lo tanto, para comprobar las propiedades de los ítems se recomienda realizar un análisis factorial exploratorio, lo que contribuye a seleccionar un conjunto de ítems homogéneos (Floyd \& Widaman, 1995).

Las escalas de medida para cada uno de los constructos quedaron conformadas como se muestra en el Cuadro 3. Sin embargo, es de resaltar que se debe procurar utilizar enfoques multidisciplinarios para validar constructos, porque eso puede remediar las deficiencias de los enfoques clásicos (Molloy, Chadwick, Ployhart, \& Golden, 2011).

\section{CUADRO 3. Constructos e Indicadores}

\begin{tabular}{|c|c|c|c|c|c|}
\hline Constructo & $\begin{array}{l}\text { Gestión del } \\
\text { Conocimiento } \\
\text { (Nonaka \& Takeuchi, } \\
\text { 1995; Villafañe, 2013) }\end{array}$ & $\begin{array}{l}\text { Marca } \\
\text { (Aaker, 2004; } \\
\text { Curubeto, 2007; } \\
\text { Capriotti, 2009) }\end{array}$ & $\begin{array}{l}\text { Reputación } \\
\text { Organizacional } \\
\text { (Fombrun, 1996; } \\
\text { Villafañe, 2005, } \\
\text { 2013) }\end{array}$ & $\begin{array}{l}\text { Responsabilidad } \\
\text { Social Empresarial } \\
\text { (Vallaeys, 2007) }\end{array}$ & $\begin{array}{l}\text { Gestión } \\
\text { Estratégica } \\
\text { (Guerra \& Navas, } \\
\text { 2008) }\end{array}$ \\
\hline Indicadores & 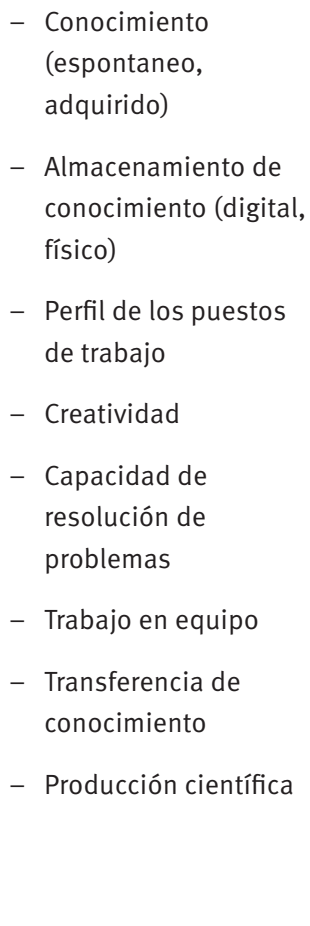 & $\begin{array}{l}\text { - Nombre oficial } \\
\text { - Logotipo y } \\
\text { Simbología } \\
\text { - Diferenciación de } \\
\text { la competencia } \\
\text { - Infraestructura } \\
\text { acorde al servicio } \\
\text { - Comunicación } \\
\text { de marca } \\
\text { institucional } \\
\text { (medios) }\end{array}$ & $\begin{array}{l}\text { - } \text { Reconocimiento de } \\
\text { - Cultura } \\
\text { organizativa } \\
\text { - } \text { Relaciones con } \\
\text { grupos de interés } \\
\text { - Oferta académica } \\
\text { reconocida } \\
\text { - } \text { Reconocimiento } \\
\text { de centros de } \\
\text { investigación } \\
\text { - Participación } \\
\text { en alianzas y } \\
\text { convenios de } \\
\text { cooperación } \\
\text { - Acreditación de la } \\
\text { oferta académica } \\
\text { - Capacidad de } \\
\text { respuesta }\end{array}$ & $\begin{array}{l}\text { - Políticas de } \\
\text { protección de medio } \\
\text { ambiente } \\
\text { _ Apoyo a sectores } \\
\text { menos favorecidos } \\
\text { - Voluntariado } \\
\text { - Investigación } \\
\text { para solucionar } \\
\text { problemas sociales } \\
\text { - Formación } \\
\text { dirigida lo social y } \\
\text { medioambiental } \\
\text { - Ética y valores }\end{array}$ & $\begin{array}{l}\text { - Análisis del } \\
\text { entorno } \\
\text { _ Integración a la } \\
\text { cadena de valor } \\
\text { - Alineación con } \\
\text { la filosofía de } \\
\text { gestión } \\
\text { - Comunicación } \\
\text { interna y externa } \\
\text { - Evaluación } \\
\text { y control de } \\
\text { recursos }\end{array}$ \\
\hline
\end{tabular}




\section{ANÁLISIS Y DISCUSIÓN DE RESULTADOS}

El análisis estadístico de los datos, se realizó en dos etapas. Primero se analizaron las propiedades del modelo de medida utilizando la técnica del análisis factorial exploratorio (AFE) y confirmatorio (AFC). A continuación se procedió a contrastar las hipótesis planteadas aplicando la técnica de estructura de covarianzas (MEE) Anderson y Gerbing (1988). La obtención de los valores de los parámetros estimados se realizó por el método de máxima verosimilitud (robusto).

Con el fin de verificar si la muestra se puede considerar homogénea, se realizaron pruebas estadísticas de comparación de medias para muestras independientes (prueba de Leneve y pruebas T). Obteniendo como resultado que las muestras son estadísticamente homogéneas para cada uno de los constructos. Por lo que se asume igualdad de varianzas.
El análisis factorial confirmó la presencia de cinco factores, cuatro de los cuales están referidos a los tipos de Recursos Intangibles y uno a la Gestión de los Recursos Intangibles. Con la finalidad de dotar al modelo de validez discriminante y convergente según lo plantea Bagozzi (1981), se procedió a eliminar los ítems con cargas no significativas menores a 0,6 y varianzas escasas menores a 50\%. A los ítems que permanecieron se les realizó un AFC. Los resultados se muestran en la tabla 1 y 2.

\section{TABLA 1. Coeficientes de correlación}

\begin{tabular}{c|c|c|c|c|c}
\hline & GC & M & RO & RS & GE \\
\hline GC & 0,87 & & & & \\
\hline M & 0,36 & 0,73 & & & \\
\hline RO & 0,74 & 0,45 & 0,85 & & \\
\hline RS & 0,22 & 0,21 & 0,34 & 0,79 & \\
\hline GE & 0,86 & 0,44 & 0,78 & 0,50 & 0,94 \\
\hline
\end{tabular}

TABLA 2. Resultados del análisis factorial confirmatorio

\begin{tabular}{|c|c|c|c|c|}
\hline Constructo & AFC & $\alpha$ de Cronbach & AVE & $\%$ carga factorial \\
\hline Gestión del Conocimiento (GC) & $\begin{array}{l}\chi 2=32,53 \mathrm{df}=12 \mathrm{RMSEA}=0.069 \mathrm{CFI}=0,95 \\
\mathrm{TLI}=0,98\end{array}$ & 0,838 & 61,11 & 0,733 \\
\hline Marca (M) & $\begin{array}{l}\chi 2=56,35 \mathrm{df}=9 \mathrm{RMSEA}=0.08 \mathrm{CFI}=0,90 \\
\mathrm{TLI}=0,92\end{array}$ & 0,868 & 53,04 & 0,684 \\
\hline $\begin{array}{l}\text { Reputación } \\
\text { Organizacional(RO) }\end{array}$ & $\begin{array}{l}\chi 2=32,59 \mathrm{df}=13 \mathrm{RMSEA}=0.072 \mathrm{CFI}=0,98 \\
\mathrm{TLI}=0,96\end{array}$ & 0,814 & 64,27 & 0,712 \\
\hline $\begin{array}{l}\text { Responsabilidad } \\
\text { Social (RS) }\end{array}$ & $\begin{array}{l}\chi 2=24,18 \mathrm{df}=8 \mathrm{RMSEA}=0.079 \mathrm{CFI}=0,91 \\
\mathrm{TLI}=0,98\end{array}$ & 0,774 & 53,63 & 0,699 \\
\hline $\begin{array}{l}\text { Gestión Estratégica } \\
\text { (GE) }\end{array}$ & $\begin{array}{l}\chi 2=10,12 \mathrm{df}=2 \mathrm{RMSEA}=0.071 \mathrm{CFI}=0,99 \\
\mathrm{TLI}=0,98\end{array}$ & 0,765 & 66,99 & 0,752 \\
\hline
\end{tabular}

Al verificar la pertinencia de los constructos se procedió a realizar el contraste de MEE. Las pruebas estadísticas para el modelo teórico inicialmente planteado, indican un ajuste razonable a los datos $\left(\chi^{2}=480,836, \mathrm{df}=133\right.$, $\mathrm{TLI}=0,81 \mathrm{CFI}=0,827 \mathrm{RMSEA}=0,097)$. Sin embargo, los resultados no apoyan todas las hipótesis del modelo, las regresiones entre los constructos Gestión Estratégica y Marca (GE - M = -0,144; $p<0,045)$ y entre Gestión Estratégica y Responsabilidad Social (GRI - RS $=-0,107$ p 0,030 ) se consideran no significativas.
Estos resultados reflejan la poca importancia que las IES le atribuyen a la marca y a la responsabilidad social. Por lo que se evidencia que las IES siguen enfocando su actuación hacia la enseñanza e investigación para generar reputación organizacional, sin lograr encajar en su gestión la vinculación con la sociedad, lo cual se refleja en los intangibles de las hipótesis 2 y 4.

Al realizar adecuaciones necesarias del modelo, que consintieron en eliminar los constructos correspondiente a la Marca y a la Responsabilidad Social, se logró un mejor ajuste del mismo, quedando especificado como lo muestra la Figura 2. 
Figura 2. Modelo final de la influencia de los tipos de Recursos Intangibles en la Gestión Estratégica de las IES.

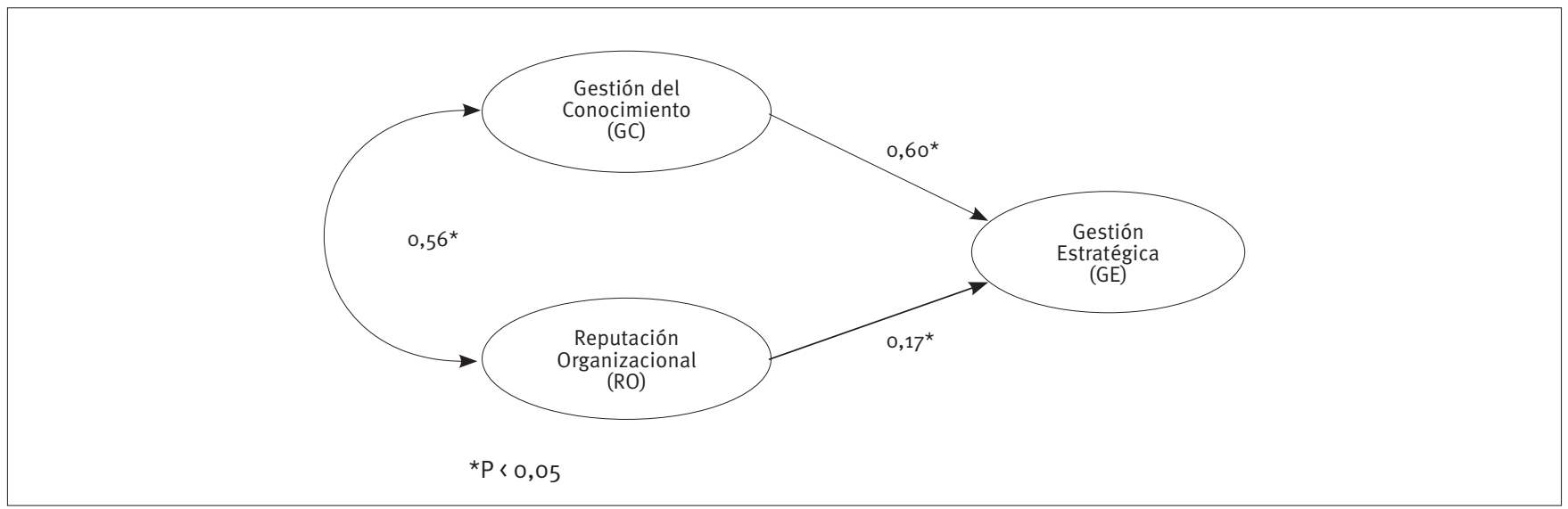

En este modelo las relaciones entre las variables sugieren que cuanto mayor es la Gestión del Conocimiento mayor es la Reputación Organizacional y por ende el nivel de inclusión de estos recursos en la gestión estratégica de las IES. Los índices de bondad del ajuste del modelo quedaron establecidos de la siguiente forma: $(x 2=214,315, \mathrm{df}=105, \mathrm{TLI}=0,921 \mathrm{CFI}=0,939 \mathrm{RMSEA}=$ 0,065), notándose de esta forma una mejora en los índices de ajuste del modelo con respecto al modelo teórico inicial.

Puede decirse que los resultados obtenidos apoyan las hipótesis $\mathrm{H}_{1} \mathrm{y}_{3}$, de modo que se puede confirmar la existencia de una relación directa y positiva entre los Recursos Intangibles Gestión del Conocimiento y Reputación Organizacional y la Ges. tión Estratégica de las IES.

\section{CONCLUSIONES}

De acuerdo a los resultados obtenidos se puede afirmar que se ha logrado el objetivo de esta investigación, que consistía en determinar la influencia ejercida por los recursos intangibles en la gestión estratégica en IES. Asimismo, se resalta la importancia de la TRC en el contexto de las IES latinoamericanas, lo que constituye un aporte a la fundamentación empírica de la TRC y de los intangibles.

Sin embargo, los resultados revelan que no todos los recursos intangibles definidos por Villafañe (2005) impactan en igual medida ni con la misma significancia. Así, los recursos Gestión del Conocimiento y Reputación Organizacional son determinantes en los procesos de gestión estratégica que realizan las IES, quedando excluida la influencia que pueden ejercer la Marca y la Responsabilidad Social.

Lo anterior se explica debido a que las IES consideran estratégicos aquellos recursos intangibles sobre los cuales ejercen mayor control. Así se tiene que la Gestión del Conocimiento es más eficaz que la Reputación Organizacional en ejercer in- fluencias en los procesos de dirección estratégica, porque las IES están enfocadas en mayor medida hacia la docencia.

Asimismo, queda demostrado que las IES no consideran relevantes las influencias que puedan ejercer los recursos intangibles que reflejan directamente la vinculación con el entorno y sobre los cuales ejercen poco control. Por lo que se concluye que los intangibles Marca y Responsabilidad Social no son considerados estratégicos de acuerdo con la definición de recurso estratégico propuesta por Barney (1991).

Por lo tanto se puede asegurar que cuanto más se profundiza en la Gestión del Conocimiento en las IES, mayor es la mejora de su Reputación Organizacional, reafirmándose de esta manera la condición de recursos estratégicos de estos intangibles.

En general, al contrastar las evidencias empíricas con las teorías sobre el tema, se puede corroborar la existencia de brechas entre ambas, como consecuencia de la no confirmación de algunas de las hipótesis. Sin embargo, el enfoque de la TRC se puede considerar adecuado para el contexto de las IES.

Se confirma también la existencia de una relación positiva entre la posesión de recursos intangibles y la gestión estratégica. No obstante, queda reflejado que en las IES no se está aprovechando el potencial estratégico de todos sus recursos intangibles. Esta relación puede mejorarse con la inclusión de los intangibles vinculados al entorno de forma tal que las IES realicen una gestión más integral, lo que demuestra la capacidad de las IES para adoptar y adaptar los principios de dirección estratégica, tal como lo afirman las investigaciones internacionales sobre el tema.

No obstante, al ser esta una investigación descriptiva, se pone de manifiesto la necesidad de seguir profundizando en el tema, apuntando esfuerzos a la medición de los constructos, por considerar que las escalas de medida construidas constituyen un aporte a este campo, pero que aún no están consolidadas. 
Asimismo, se debe aplicar el estudio en muestras de mayor tamaño para obtener relaciones más significativas entre las variables y realizar ajustes del modelo considerando cualquier otra variable que pueda influir en los procesos de gestión de recursos intangibles en las IES.

\section{REFERENCIAS}

Aaker D. (2004). Brand portfolio strategy. Creating relevance, differentiation, energy, leverage and clarity. Free Press, New York.

Accenture. (2012). El valor de los intangibles para la financiación de las PYMEs. Catedra UAM-Accenture en Economía y Gestión de la Innovación, Madrid.

Alvarado, A. \& Schlesinger, M. (2008). Dimensionalidad de la responsabilidad social empresarial percibida y sus efectos sobre la imagen y la reputación: Una aproximación desde el modelo de Carroll. Estudios Gerenciales, 24(108), 37-59.

Ambrosini, V. \& Bowman, C. (2001). Tacit knowledge: Some suggestions for operationalization. Journal of Management Studies, 38(6), 811-829.

Anderson, J. \& Gerbing, D. (1988). Structural equation modeling in practice: A review and recommended two-step approach. Psychological Bulletin, 103(3), 411-423

Andriessen, D. (2004). Making sense of intellectual capital. PhD thesis, Nyenrodem University, The Netherlands. Butterworth-Heinemann, Burlington, MA.

Bagozzi, R. (1981). Evaluating structural equation models with unobservable variables and measurement error: A comment. Journal of Marketing Research, 18(3), 375-381.

Barney, J. (1991). Firm resources and sustained competitive advantage. Journal of Management, 17(1), 99-120.

Bontis, N. (1998). Intellectual capital: An exploratory study that develops measures and model. Management Decision, 36(2), 63-76.

Bricall, J. (2000). Informe Universidad 2000, Estudio encargado por la Conferencia de Rectores de Universidades Españolas (CRUE). Recuperado en 17 Mayo 2014 de www.da.montes.upm.es/docs/bricall/bricall. htm

Brooking, A. (1996). Intellectual capital: Core asset for the third millennium enterprise, International Thompson Business Press, London.

Bueno, E. (1998). El capital intangible como clave estratégica en la competencia actual. Boletín de estudios Económicos, Vol LII, Madrid, España.

Bueno, E. (2003). Enfoques principales y tendencias en dirección del conocimiento (Knowledge Management), en R. Hernández (ed.): Dirección del Conocimiento: Desarrollos teóricos y aplicaciones, Ediciones la Coria, Trujillo (Cáceres).

Bustos, E. (2007). La Gestión del Capital intelectual en las Instituciones de Educación Superior, ante el reto de la innovación en la sociedad del conocimiento. Administración Contemporánea. Revista de Investigación. 1(7), 1-23

Cañibano, L., García-Ayuso, M., \& Sánchez, M. (1999). La relevancia de los intangibles para la valoración y la gestión de empresas: revisión de la literatura. Revista Española de Financiación y Contabilidad, (100), 17-88.
Cañibano, L., Sánchez, P., García-Ayuso, M, \& Chaminade, C. (eds). (2002). Meritum Guidelines for Maaging and Reporting on Intangibles, en, Fundarción Airtel Móvil, Madrid.

Cañibano, L. \& Sánchez, M. (2004). Medición, gestión e información de intangibles: lo más nuevo. Revista de Contabilidad y Dirección, 1, 99-139.

Cañibano, L. \& Sánchez, M. (2008). Intellectual capital management and reporting in universities and research institutions. Estudios de economía aplicada, 26(2), 7-26.

Cañibano, L. \& Sánchez, M. (2009). Intangibles in universities: current challenges for measuring and reporting Journal of Human Resource Costing \& Acconunting, 13(2), 93-104.

Capriotti, P. (2007). Gestión de la marca corporativa. La Crujía, Buenos Aires, Argentina.

Capriotti, P. (2009). Branding corporativo: Fundamentos para la gestión estratégica de la identidad corporativa. Colección libros de la empresa. Santiago, Chile.

Carretero-Dios, H. \& Pérez, C. (2005). Normas para el desarrollo y revisión de estudios instrumentales. International Journal of Clinical and Health Psychology, 5(3), 521-551.

Clark, L. \& Watson, D. (2003). Constructing validity: Basic issues in objective scale development. En A.E. Kazdin (Ed.), Methodological issues $\&$ strategies in clinical research ( $3^{\underline{a}}$ ed.) (pp. 207-231). Washington: APA.

Curubeto, C. (2007). La marca universitaria, atributos, beneficios o valores: ¿Qué utilizan las universidades de Buenos Aires para construir su identidad de marca y su propuesta de valor? ${ }^{1}$ a ed. Universidad Austral. Facultad de Comunicación. Editorial Dunken, Buenos Aires. pp. 706.

Dahlsrud, A. (2008). How corporate social responsibility is defined: An analysis of 37 definitions. Corporate Social Responsibility and Environmental Management, 15(1), 1-13.

De Gregori, W. (2002). Capital Intelectual. McGraw Hill Bogotá.

Diefenbach, T. (2006). Intangible resources: A categorial system of knowledge and other intangible assets. Journal of Intellectual Capital, 7(3), 406-420.

Dowling, G. (1994). Corporate reputations: Strategies for developing the corporate brand. London: Kogan Page.

Edvinsson, L. \& Malone, M. (1997). Intellectual capital: Realizing your company's true value by finding its hidden roots, HarperCollins Publishers, Inc., New York.

European Commission Ricardis. (2006). Report Intellectual Capital to augment research, development y innovation in SMEs. Recuperado en 25 Marzo 2013 de http://ec.europa.ec/invest-in-research/pdf/download_en/2006-2977_web1.pdf.

European Commission Innova. (2011). Recuperado en 25 Marzo 2013 de http://www.europeinnova.eu/web/guest/home//journal_ content/56/10136/21462.

Floyd, F. \& Widaman, K. (1995). Factor analysis in the development and refinement of clinical assessment instruments. Psychological Assessment, 7(3), 286-299.

Fombrun, C. (1996). Reputation: Realizing value from the corporate image. Harvard Business School Press, Boston.

Funes, Y. (2010). Valuación de los activos intangibles. Caso de la UNAM. Revista del Centro de Investigación. Universidad La Salle, 9(33), 45-60. 
Gacel-Ávila, J. (2011). Impacto del proceso de Bolonia en la Educación Superior de América Latina. En: Globalización e internacionalización de la educación superior [monográfico en línea]. Revista de Universidad y Sociedad del Conocimiento (RUSC), 8(2), 123-134. Recuperado en 12 Marzo 2013 De http://rusc.uoc.edu/ojs/index.php/rusc/article/view/ v8n2-gacel/v8n2-gacel.

Galbreath, J. \& Galvin, P. (2006). Accounting for performance variation: how important are intangible resources?. International Journal of Organizacional analysis, 14(2), 150-170.

Grant, R. (1991). The resource-based theory of competitive advantage: Implications for strategy formulation. California Management Review, 33(3), 114-135.

Grant, R. (1996). Dirección estratégica: Conceptos, técnicas y aplicaciones, Civitas, Madrid.

Guerras, L. \& Navas, J. (2008). Dirección Estratégica de la Empresa. Civitas, $4^{\underline{a}}$ ed. Madrid.

Hill, R. (2004). The socially responsible university: Talking the talk while walking the walk in the College of Business. Journal of Academic Ethics, 2(1), 89-100.

Itami, H. (1987). Mobilizing invisible assets. Harvard University Press: Cambridge, Massachusetts, Estados Unidos.

Kaplan, R. \& Norton D. (2004). Measuring the strategic readiness of intangibles assets. Harvard Business review, 82(2), 52-63.

Kaplan, R. \& Norton D. (2007). Using the Balanced Scorecard as a Strategic Management System. Harvard Business Review 1-14

Kaufmann, L. \& Schneider, Y. (2004). Intangibles: A synthesis of current research. Journal of Intellectual Capital, 5(3) 366-388.

Kotler, P. (2000). Marketing Management. The Millennium Edition, New Jersey Upper Saddle River, Prentice Hall.

Kristandl, G. \& Bontis, N. (2007). Constructing a definition for intangibles using the resource based view of the firm. Management Decision, 45(9), 1510-1524.

Krugman, P. (2012). ¡Acabad ya con esta crisis!. Barcelona Editorial Crítica.

Leitner, K. (2004). Intellectual capital reporting for universities: Conceptual background and application for Austrian universities. Research Evaluation, 1(2), 129-140.

Lev, B. (2003). Intangibles: Medición, Gestión e Información. Barcelona: Deusto.

López, M. (2012). Gerencia: Capital intelectual y sus competencias en instituciones educativas. Observatorio Laboral Revista Venezolana 5(9), 109-127.

Molloy, J. C., Chadwick, C., Ployhart, R., \& Golden, S. (2011). Making intangibles "tangible" in tests of resource-based theory: A multidisciplinary construct validation approach. Journal of Management, 37(5), 1496-1518.

Morales, V., Medina, E., \& Álvarez, N. (2003). La educación superior en Venezuela. Informe 2002 a IESALC- UNESCO. Caracas.

Nomen, E. (2005). El valor razonable de los actives intangibles: El efecto mariposa de la deslocalización. Ediciones Deusto: Barcelona.

Nonaka I. \& Takeuchi, H. (1995). The knowledge-creating company: How Japanese companies create the dynamics of innovation. Oxford University Press: New York, pp. 15-23.

Nunnally, J. \& Bernstein, I. (1995). Teoría psicométrica. Madrid: McGraw-Hill.
OECD. (2010). A new OECD project: New sources of growth: Intangible assets. Recuperado en 25 Agosto 2011 de http://www.oecd.org/dataoecd/60/40/46349020.pdf. a

Petty, R. \& Guthrie, J. (2000). Intellectual capital literature review: Measurement, reporting and management. Journal of Intellectual Capital, 1(2), 155-176.

Rodríguez-Ponce, E., Pedraja-Rejas, L., Araneda-Guirriman, C., \& Rodríguez-Ponce, J. (2013). La relación entre la gestión del conocimiento y la gestión académica: un estudio exploratorio en universidades chilenas. Interciencia, 38(2), 88-94.

Roos, J., Roos, G., Edvinson, L., \& Dragonetti, N. (1998). Intellectual capital. navigating in the new business landscape. New York University Press: New York.

Saíz-Bárcena, L, \& Díez-Pérez, J., Manzanedo Del Campo, M., \& Rodríguez-Monroy, C. (2013). Intercambio del conocimiento en la empresa: Aprendiendo de la experiencia. Interciencia, 38(8), 570-576.

Sánchez, P. (2008). Papel de los intangibles y el capital intelectual en la creación y difusión del conocimiento en las organizaciones. Situación actual y retos de futuro. ARBOR Ciencia, pensamiento y Cultura, $732 \mathrm{Ou}$ lio-Agosto), 575-594.

Sánchez, P., Elena, S., \& Castrillo, R. (2006). Intellectual capital management and reporting for universities: The case study of the Autonomous University of Madrid. paper presented at the 2nd Workshop on Visualising, Measuring and Managing Intangibles and Intellectual Capital, Maastricht, The Netherlands, 25-27 October.

Sanchez, R. \& Heene, A. (2004). The new strategic management: Organization, competition and competence. John Wiley \& Sons: Chichester.

Secundo, G., Margherita, A., Elia, G., \& Passiante, G. (2010). Intangible assets in higher education and research: mission, performance or both? Journal of Intellectual Capital. 11(2), 140-157.

Seemann, P., Delong, D., Stucky, S., \& Guthiere, E. (2000). Building Intangible Assets: A strategic framework for investing in intellectual capital. Knowledge Management: Classic and Contemporary Works, the MIT Press: Cambridge, MA, pp. 85-98.

Smith, G., Fischer, S., \& Fister, S. (2003). Incremental validity principles in test construction. Psychological Assessment, 15(4), 467-477.

Sveiby, K. (1997). La nueva riqueza organizativa, lo Berret - Koehler, San Francisco, CA.

Sveiby, K. (2010). Methods for measuring intangible assets. Recuperado en 12 Agosto 2013 de http://www.sveiby.com/articles/IntangibleMethods.htm.

Unesco. (1998). Declaración mundial sobre la educación superior en el siglo XXI: Visión y acción.

Vallaeys, F. (2007). Breve marco teórico de responsabilidad social universitaria. Recuperado en 14.09.2013 de www.usc.edu.co/extension/ index.php?option...id.

Varela-Candamio L. \& García-Álvarez M. (2012). Analysis of information and communication technologies in higher education: A case study of business degree. International Journal of Engineering Education, 28(6), 1301-1308.

Viedma J. (2007). In search of an intellectual capital comprehensive theory. Electronic Journal of Knowledge Management, 5(2), 245-256.

Villafañe, J. (2003). La buena Reputación: Claves del valor intangible de las empresas. Madrid Pirámide. 
Villafañe, J. (2005). La gestión de los intangibles empresariales. Comunicacão e Sociedade, 8, 101-103. Recuperado en 12 Octubre 2012 de http://www.villafane.com/.

Villafañe, J. (2013). La reputación de las universidades: Clave para su gestión. Conferencia en la Universidad de la Rioja, España, Octubre, 2013.

Villafañe \& Asociados. (2013). Marca Recuperado en 12.11.2013 de http:// www.villafane.com/formacion/Curso-online-reputacion-marca-6.
Villalonga, B. (2004). Intangible resources, Tobin's q, and sustainability of performance differences. Journal of Economic Behavior and Organization, 54(2), 205-230.

Wernerfelt, B. (1984). A resource-based view of the firm. Strategic Management Journal, 5(2), 171-180.

World Bank. (2011). Knowledge assessment methodology: Knowledge for development (K4D) Recuperado en 12 Septiembre 2013 en: http:// siteresources.worldbank.org/INTUNIKAM/Resources/KAMbooklet.pdf. 